\title{
AN ADDITIVE REPRESENTATION FOR REAL FUNCTIONS ON THE PRODUCT OF A SET AND A LATTICE ${ }^{1}$
}

\author{
W. J. R. EPLETT
}

\begin{abstract}
Given a real-valued function defined on the product of an arbitrary set and a finite lattice, a necessary and sufficient condition is obtained for the existence of an additive representation for the function in terms of functions on sublattices of the original lattice. This additive representation is of the nature of a recurrence and provides a tool for further analysis of the function. An example is given for a case where the lattice concerned is the lattice of partitions of a finite set. The main theorem of this paper generalizes a result due to Fishburn corresponding to the lattice being the lattice of subsets of a finite set.
\end{abstract}

1. Introduction. Suppose that $X_{1}, \ldots, X_{m}$ are $m$ nonempty (arbitrary) sets, $I_{1}, \ldots, I_{n}$ are nonempty subsets of $\{1, \ldots, m\}$ and $g$ is a real-valued function on the product set $X=\Pi_{i=1}^{m} X_{i}$. The number of elements in a finite set $S$ will be denoted by $|S|$. Fishburn [1] has proved the following result.

EXAMPLE 1. There exist real-valued functions $g_{j}$ on $\Pi_{i \in I_{j}} X_{i}, 1<j<n$, such that for each $x \in X$

$$
g(x)=\sum_{j=1}^{n} g_{j}\left(x^{j}\right),
$$

where $x^{j}=\left(x_{i}\right)_{i \in J}$, if and only if for each $x \in X$

$$
g(x)+\sum_{S}(-1)^{|S|} g\left(x\left[\bigcap_{j \in S} I_{j}\right]\right)=0
$$

where $x[I]$ is defined in terms of some fixed $x^{0} \in X$ as the $m$-tuple whose $i$ th coordinate is $x_{i}$ if $i \in I$ and $x_{i}^{0}$ if $i \notin I$ and the summation in (2) is over nonempty $S \subset\{1, \ldots, n\}$.

It is the purpose of this note to generalize Example 1. Notice that (2) involves summation over members of the lattice of subsets of $\{1, \ldots, n\}$ and also uses the Möbius function for that lattice. This suggests the possibility of generalizing Fishburn's result to cover other lattices.

2. The additive representation theorem. Suppose that $\mathcal{L}$ is a finite lattice having a least element 0 and let us denote by $\mathcal{Q}$ the set of atoms (or points) of $\mathcal{L}$. Let $\mathscr{X}$ be an arbitrary set. The real-valued function $f$ on $\mathcal{X} \times \mathcal{L}$ is said to have the interchange property under $P$ if there exists a mapping $P$ : $\mathfrak{X} \times \mathfrak{L} \rightarrow \mathfrak{X}$ which for each $x \in \mathscr{X}$ and $z \in \mathcal{L}$ takes $(x, z)$ to $x_{z} \in \mathcal{X}$ in such a way that

Received by the editors June 19, 1978.

AMS (MOS) subject classifications (1970). Primary 06A15.

${ }^{1}$ Research supported by a British Council Research Award. 


$$
f(x, z \vee y)=f\left(x_{z}, y\right)=f\left(x_{(z \vee y)}, 0\right)
$$

for all $y \in \mathcal{L}$. In particular, therefore, $f(x, z)=f\left(x_{z}, 0\right)$.

For $a \in \mathbb{Q}$ define the sublattice $\mathfrak{L}_{a}$ as the elements of $\mathcal{E}$ which are of the form $z \vee a(z \in \mathcal{L})$. The following theorem about functions with the interchange property can be proved.

THEOREM 2. For each $x \in \mathcal{X}, z \in \mathcal{L}$, the function $f$ having the interchange property under $P$ can be expressed as

$$
f(x, z)=\sum_{a \in \mathbb{Q}} f_{a}(x, z \vee a)
$$

where for each $a \in \mathbb{Q}, f_{a}$ is a real-valued function on $\mathcal{X} \times \mathfrak{L}_{a}$ satisfying the interchange property under $P_{a}$, the restriction of $P$ to $\mathscr{X} \times \mathfrak{L}_{a}$, if and only if for each $x \in \mathfrak{X}$

$$
\sum_{z>0} \mu(0, z) f(x, z)=0
$$

where $\mu$ is the Möbius function of the lattice $\mathcal{L}$ (as defined in Rota [2]).

Proof. Suppose that (4) is true. Then, for given $x \in \mathcal{X}$ and $a \in \mathcal{Q}$,

$$
\sum_{z>0} \mu(0, z) f_{a}(x, z \vee a)=\sum_{y \in \mathfrak{E}_{a}} f_{a}(x, y) \sum_{z \vee a=y} \mu(0, z)=0
$$

because $\Sigma_{z \vee a=y} \mu(0, z)=0$ by the corollary to Proposition 4 of $\$ 5$ of [2]. Clearly (5) now follows from (4).

Suppose that (5) holds. Define a rank function for $\mathscr{Q}$ by a bijection $r$ mapping $\mathscr{Q}$ to $\{1, \ldots,|\mathbb{Q}|\}$. For each $a \in \mathbb{Q}$ define

$$
\mathcal{S}_{a}=\{z \in \mathcal{L}: z \geqslant a \text { and if } z \geqslant b, b \in \mathbb{Q} \text {, then } r(b)<r(a)\} .
$$

These are disjoint sets whose union is $\mathfrak{L} \backslash\{0\}$. Now, by (5), for each $x \in \mathcal{X}$

$$
f(x, 0)=-\sum_{y>0} \mu(0, y) f(x, y)=-\sum_{a \in \mathfrak{Q}} \sum_{y \in \delta_{a}} \mu(0, y) f(x, y),
$$

so that putting $f_{a}(x, a)=-\Sigma_{y \in \delta_{a}} \mu(0, y) f(x, y)$, (4) will be satisfied when $z=0$.

If $z \in \mathcal{L}$, then $f_{a}(x, z \vee a)$ can be defined by

$$
f_{a}(x, z \vee a)=-\sum_{y \in \delta_{a}} \mu(0, y) f\left(x_{z}, y\right) .
$$

In order to verify that $f_{a}$ is a well-defined function on $\mathfrak{X} \times \mathfrak{E}_{a}$, we have to show that if $u \vee a=v \vee a$, then $f_{a}(x, u \vee a)=f_{a}(x, v \vee a)$. However,

$$
\begin{aligned}
f_{a}(x, u \vee a) & =-\sum_{y \in \delta_{a}} \mu(0, y) f\left(x_{u}, y\right)=-\sum_{y \in \delta_{a}} \mu(0, y) f(x, u \vee y) \\
& =-\sum_{y \in \delta_{a}} \mu(0, y) f(x, v \vee y)
\end{aligned}
$$

(because $y \geqslant a$, it follows that $u \vee y=v \vee y$ )

$$
=f_{a}(x, v \vee a) \text {, }
$$

so that $f_{a}$ is indeed well defined. The additive representation (4) follows from (3) 
and (6). The definition of the functions $f_{a}, a \in \mathbb{Q}$, ensures that for $x \in \mathscr{X}$ and $y, z \in \mathcal{L}_{a}$ the relationship $f_{a}(x, y \vee z)=f_{a}\left(x_{z}, y\right)=f_{a}\left(x_{(y \vee z)}, a\right)$ holds. This completes the proof of the theorem.

Some further comments about the theorem seem appropriate. The additive representation we have described in Theorem 2 has the form of a recurrence. Each function $f_{a}(a \in \mathcal{Q})$ is defined on a sublattice of $\mathcal{E}$ and this function also has the interchange property. So the functions used in the representation may have an additive representation in their turn.

As demonstrated in [1] for Example 1, the functions $f_{a}$ defined in the proof of Theorem 2 are not necessarily the only ones for which (4) holds. In the light of the remarks of the preceding paragraph they are, however, particularly appropriate.

Finally notice that we could without difficulty alter lattice to semilattice throughout the discussion.

3. Applications of the theorem. First we shall verify that Example 1 is indeed a special case of Theorem 2 . In the statement of the theorem, take $\mathscr{X}=X=\prod_{i=1}^{m} X_{i}$ and $\mathcal{L}$ as the lattice of subsets of $\{1, \ldots, n\}$ ordered by inclusion with the join operation defined by union of sets. The atoms of $\mathcal{L}$ are the sets $\{i\}, 1 \leqslant i \leqslant n$.

If $g$ is a real-valued function on $X$, then define $f(x, z)=g\left(x\left[\cap_{j \in z} I_{j}\right]\right)$ for $z \neq \varnothing$ (the empty set) and $f(x, \varnothing)=g(x)$. Then (3) holds for $f(x, z)$ with $x_{z}=x\left[\cap_{j \in z} I_{j}\right]$ $(z \neq \varnothing)$ and $x_{\varnothing}=x$. Fishburn's result is obtained from Theorem 2 by defining $g_{j}\left(x^{j}\right)=f_{j}$ where $\bar{j}=\{j\}, 1 \leqslant j \leqslant n$. For this to be a projection it must satisfy the condition that if $x\left[I_{j}\right]=y\left[I_{j}\right]$, then $f_{j}(x, \bar{j})=f_{j}(y, \bar{j})$. This condition is satisfied because $f_{j}(x, \bar{j})=f_{j}\left(x_{\bar{j}}, \bar{j}\right)=f_{j}\left(x\left[I_{j}\right], \bar{j}\right)$.

As a novel application of Theorem 2-although the example is constructed along similar lines to Example 1-let us take $\mathcal{L}$ to be the lattice $\Pi_{n}$ of partitions of the set $\{1, \ldots, n\}$. The partitions are ordered by refinement with the join of two partitions defined as the least partition of which $\Pi_{1}$ and $\Pi_{2}$ are both refinements. The partition consisting of $n$ singletons is then the least element of the lattice.

Consider a function $x$ which maps the blocks of some partition in $\Pi_{n}$ to the real numbers. The class of such functions will be denoted by $\mathscr{X}$ and the partition mapped by a particular $x \in \mathcal{X}$ will be denoted by $\Pi(x)$. The sum of $x \in \mathfrak{X}$ over $\sigma \in \Pi_{n}$ is defined to be the function $\bar{x}_{\sigma} \in \mathcal{X}$ which maps the blocks of $\sigma \vee \Pi(x)$ to the real numbers as follows:

$$
\begin{aligned}
& \text { if } B(\sigma \vee \Pi(x)) \text { denotes a block of } \sigma \vee \Pi(x) \text {, then } \bar{x}_{\sigma}(B(\sigma \vee \Pi(x)) \\
& =\Sigma_{\tau \in \delta_{B}} x(\tau)
\end{aligned}
$$

where $\mathcal{S}_{B}=\{$ blocks of $\Pi(x)$ contained in $B(\sigma \vee \Pi(x))\}$.

If $g$ is a real-valued function on $\mathcal{X}$, then define $f(x, \sigma)=g\left(\bar{x}_{\sigma}\right)$ for $x \in \mathcal{X}$, $\sigma \in \Pi_{n}$. It is easy to verify that $f$ defined in this way has the interchange property and so necessary and sufficient conditions for an additive representation for $f(x, \sigma)$ may be obtained from Theorem 2 . The atoms of $\Pi_{n}$ are the $\frac{1}{2} n(n-1)$ partitions consisting of a single block containing two elements with the remaining blocks being singletons. The Möbius function for $\Pi_{n}$ required by condition (5) of Theorem 2 is given explicitly in [2] and will not be repeated here. 
EXAMPLE 3. The representation of $f(x, \sigma)$ obtained from Theorem 2 can be written as

$$
f(x, \sigma)=\sum_{1<i<j<n} f_{i j}\left(x, \sigma^{i j}\right),
$$

where $\sigma^{i j}$ is the partition of a set of $n-1$ elements obtained by combining the blocks of $\sigma$ containing $i$ and $j$ (if they are in different blocks) and identifying $i$ and $j$. The functions on the right-hand side of (7) are therefore essentially functions on $\Pi_{n-1}$, providing a recurrence with which to study $f$.

\section{REFERENCES}

1. P. C. Fishburn, Additive representations of real functions on product sets, J. Combinatorial Theory 4 (1968), 397-402.

2. G. C. Rota, On the foundations of combinatorial theory. I, Theory of Möbius functions, $\mathbf{Z}$. Wahrscheinlichkeitstheorie und Verw. Gebiete 2 (1964), 340-368.

Department of Mathematics, University of Reading, Whiteknights, Reading RG6 2aX ENGLAND

Current address: Mathematical Institute, University of Oxford, 24-29 St. Giles, Oxford OX1 3LB, England 